\title{
Experiences in the implementation of a national policy: A retrospective \\ analysis of the Australian Chronic Dental Disease Scheme
}

\author{
Raymond Lam, Estie Kruger, Marc Tennant
}

Centre of Rural and Remote Oral Health, The University of Western Australia

\section{RESEARCH}

Please cite this paper as: Lam R, Kruger E, Tennant $M$. Experiences in the Implementation of a national policy: $A$ retrospective analysis of the Australian Chronic Dental Disease Scheme. AMJ 2012, 5, 10, 551-559. http://doi.org/10.21767/AMJ.2012.1479

\section{Corresponding Author:}

Estie Kruger

Centre for Rural and Remote Oral Health

The University of Western Australia (M498)

35 Stirling Highway

CRAWLEY WA 6009

Australia

Email: ekruger@crroh.uwa.edu.au

\section{Abstract}

\section{Background}

The Chronic Dental Disease Scheme (CDSS) is the first public dental policy in Australia to attract Medicare benefits for dental services.

\section{Aims}

This study examines the utilisation of a new federal method of funding dental care in Australia and provides an insight into the implications of government dental programs. The program titled; Chronic Dental Disease Scheme, provided government--subsidised dental care for people suffering from a chronic medical condition.

\section{Method}

A retrospective analysis of activity data using the relevant item numbers were extracted from the open source Medicare Benefits Schedule database (MBS) for years 2007--2009.

Results

During the study period, a total of approximately five million dental services were provided. There was a disproportionate use of services between jurisdictions. The highest proportion $(66 \%)$ of services was provided in the state of New South Wales (NSW) with Victoria second (22\%). The adjusted value of care provided as a proportion of comprehensive examinations ranged from $\$ 1937$ in the northern territory (NT) to $\$ 2900$ in NSW. The value of care per dentist ranged from nearly $\$ 80000$ down to less than $\$ 1000$ and the value of care per adult of the population ranged between $\$ 53$ and

$\$ 1$ across Australia. The highest was always in NSW and the lowest always being the NT. Fixed prosthodontics (reconstruction) accounted for the significant costs associated with the program.

Conclusion

The scheme has been utilised above its budget estimate with prosthodontics accounting for the majority of expenses. Treatment plans differed between jurisdictions. The increase in utilisation of the scheme was coincident with periods of increased in subsidy and remuneration and has been postulated to be a main driver for its utilisation rather than the improvement in chronic health.

Key Words

Dental Public Health, Computational Mathematics, Monte Carlo

\section{What this study adds:}

1. The Chronic Dental Disease Scheme (CDSS) is the most expensive and controversial public dental policy in Australian history.

2. An analysis of the CDSS provides an unprecedented opportunity to analyse a national dataset of dental policy in practice at the epidemiological level.

3. This study provides an invaluable insight into the design and implementation of dental policy in Australia.

\section{Background}

One of the hallmarks of a developed country is a reasonable standard of living and health. There is a general expectation that the government of a developed country has the responsibility to provide adequate health care services for its population. Australia is a federated system of government and is generally considered to be a developed country. Epidemiological studies have indicated that Australia has one of the healthiest populations in the world with a life expectancy considered to be enviable to most other countries ${ }^{1}$. However, an increasingly aging population brings 
complications to the health care system. It has been estimated that over one-third of problems in medicine are chronic in nature, are responsible for over $80 \%$ of the burden of disease and injury and continually form a significant part of health expenditure. ${ }^{2,3}$

Australia's national health insurance scheme, Medicare, is funded by annual taxation and is designed to provide health services for its population. One of the defining features of this system is bulk billing where the costs of the health service are funded by the government without the patient incurring cost for treatment. Typically, the health provider itemises the treatment and directly bills the government agency responsible for the administration of the service (Australian Department of Human Resources). Despite government funding, health providers are able to set their own fees for services provided and patients are often required to pay a contribution of the costs above the level of subsidy set by the government. Therefore bulk billing is not always guaranteed under this system and patients are often required to make a co-contribution which may influence acceptance of non-acute services that are cosmetic or elective. Around $76 \%$ of Medicare rebatable services are currently bulk billed. ${ }^{4,5}$

Despite being a universal health care scheme whereby eligibility for assistance is available to all regardless of income or health status, there are limits to the scope of health under this system. Contrary to the view expressed by the World Health Organization, oral health is not considered to be part of general health by virtue of its omission from the Medicare system. ${ }^{6}$ Diseases in the mouth receive no government assistance whilst organs within this vicinity such as the ear, nose, neck and throat are recognised under Medicare. Ironically, oral diseases in the form of caries and periodontal disease are the most prevalent forms of disease within the community and is the second most costly diet-.. related disease with an economic impact comparable to heart disease and diabetes. ${ }^{7}$ With minimal government assistance, the majority of dental expenses are borne by the individual. Over $85 \%$ of Australian dental care is provided by private practices on a fee-for--service basis with health insurance coverage of about $40 \%$ of the population. A survey of dental fees in Australia indicates that private dental fees have increased substantially higher than the Consumer Price Index and other health services making access to dentistry less accessible to the population. It has been estimated that at least one--third of Australians delay or avoid seeking dental services due to cost. ${ }^{8}$

Since the Medicare system was implemented by the Commonwealth government in 1984, debate over funding for dentistry under this system has continued to remain unresolved. A number of inquiries had considered the inclusion of dentistry under Medicare including the Layton inquiry in 1986 and two separate senate inquires in 1998 and $2003 .{ }^{9}$ On each occasion, dentistry was excluded from Medicare despite repeated calls from oral health associations around the country that oral health was integral to general health. Much of the debate centered around the potentially high cost of including dentistry under Medicare rather than debating the association between oral and general health, which evidenced=based dentistry continues to indicate a correlation. It must be noted that although Medicare excluded dentistry from Medicare, government assistance was provided to a small cohort of the population such as cleft lip and palate patients (incidence 1:700 births) and war veterans under the Department of Veterans Affairs program.

In 2004, the Coalition Liberal Government introduced Medicare benefits for a limited range of dental services to treat patients with chronic and complex diseases. Known as the Enhanced Primary Dental Care Scheme (EPDCS), this scheme formed part of a larger initiative, the Enhanced Primary Care Scheme (EPC). The EPDCS represented a deviation from previous governments in that this was the first community--based dental program to be subsidised by Medicare benefits, albeit in a specified form. Dentistry was not the only health profession that received government assistance as a range of other allied health professions such as podiatry, physiotherapy and audiology were included under the EPC, although under a separate sub-program. Under the EPC schemes, dentistry and the allied health professions required a management plan formulated by the general medical practitioner (GMP) prior to receiving subsidy for treatment. In this manner, the GP was the chief architect of the EPC. Eligibility for treatment was based on any condition lasting for six months or longer as acknowledged by the GMP with no other eligibility criteria or income test.

The utilisation of the EPDCS was extremely low with a combined number of services during the years 2004-2007 totalling 16,000 services nationally. ${ }^{10}$ With concerns that costs involved with administration of the program would outweigh any benefits from the program due to poor utilisation of the scheme, the scope of treatment and subsidy of the program was increased significantly on two occasions. This ultimately resulted in the subsidy set at $\$ 4250$ over two years with the full range of dental services available. With these changes, the program was rebranded as the CDSS.

With a budget forecast of $\$ 385$ million over four years, 
claims to the Department of Health that administered the CDSS surmounted to over one billion dollars annually making it the most expensive dental policy in Australian history. The number of dental service items increased from 16,000 to over five million. In contrast to initial teething problems with underutilisation, cost containment presented as a significant issue as the sustainability of the program was at risk. This resulted in repeated attempts to remove the program to direct funding to another Commonwealth government initiative (Commonwealth Dental Health Plan) only to result in the legislation being blocked in the senate by the minor parties. Other reactionary measures included an aggressive auditing campaign by Medicare. This involved intensive scrutiny of claims which resulted in practitioners required to repay significant sums due to inappropriate conduct such as claiming for treatment not provided, failing to formulate an appropriate treatment plan and other administration deficiencies. These problems drew widespread participation by influential stakeholders including all political parties, the media, Australian Dental Association and a variety of oral health associations and these exchanges were often heated with blame shifting between the groups as a unified and effective policy remains elusive. Consequently, the future of the CDSS remains in limbo as there is no general consensus to the way forward for public dentistry in Australia. Central to this problem is that research in Australian dental public health is minimal and has not kept pace with other areas of dental science. It is possible that this void in knowledge or ambiguity had led to continual disagreement between influential bodies responsible for oral health.

A study of the CDSS would provide an invaluable contribution to oral health policy. It is rare to be able to study dental health policy in practice at the epidemiological level and a study of this scale is unprecedented in Australia. This study also involved unprecedented action by Medicare in its audit to pursue payments involved with the scheme. As the CDSS is the most expensive dental initiative in Australian history and given that the search for a direction for the future of dental policy in Australia is unclear, the CDSS may provide important clues to the direction forward. Research would also be applicable internationally as many countries aspire to replicate the enviable life expectancy enjoyed by Australians and there is no doubt that oral health would play a key role to this outcome. With this in mind, the aim of this study was to examine the use of the CDSS across Australia to provide insights into the delivery of government subsidised dental programs and factors that influence utilisation. The scope of this study has been limited to the jurisdictional level with analysis of the six states and two territories in Australia. As the EPC Medicare dental scheme began as the EPDCS, a brief mention of the utilisation of this scheme will provide a context in which to analyse the CDSS.

\section{Method}

Base activity data:

All data was obtained from open sources and therefore no ethics was required for the study. ${ }^{11}$ Specifically, the department responsible for processing all Medicare claims lodged by practitioners (Department of Human Resources, Australian Government) provided a public assessable electronic database (Medicare Benefits Schedule Database) of these services under the categories of jurisdiction, age and gender. Staff employed by the department was responsible for data collation and this study assumed that no external party influenced the manner in which data was recorded.

Consistent in general practice dentistry in Australia with regards to itemising treatment, this study refers to dental services as a single act of dentistry. Based on the Australian Schedule of Dental Services and Glossary, ${ }^{12}$ each dental service was categorised into 10 main facets of dentistry: (i) diagnostic, (ii) preventive, (iii) periodontics, (iv) oral surgery, (v) endodontic, (vi) restorative, (vii) prosthodontic, (dentures), (viii) crown and bridge, (ix) orthodontic, (x) general.

As the focus of this study was to analyse patterns of dental services in general practice dentistry, data related to specific groups that limited their scope of practice such as prothesists (limited to dentures) and the specialties (limited to their area of expertise) were removed from the dataset before analysis. Data pertaining to Medicare items related to dentistry (85011-87777) were extracted from the Medicare Benefits Schedule database (MBS) online, exported and analysed in Excel 97 (Microsoft Corp Redmont WA) for the calendar years 2007-2009 during the operation of the CDSS. Baseline data from the utilisation of the original EPDCS was given to provide an initial comparison. Comparison between the two schemes would highlight the influences of remuneration schedules in the intensity of treatment. As the national dataset of treatment represented actual utilisation patterns of over five million service items, this study did not rely on inferential statistics or errors associated with sampling and forecasting. Conclusions were derived directly from observation of tables and figures derived from the data.

Population of value of care: Population--based comparisons were made based on data obtained from the 2009 Australian Bureau of Statistics. ${ }^{13}$ Dentist numbers for each jurisdiction were obtained from the Australian Institute of Health and Welfare. ${ }^{14}$ Value of care calculations (adjusted 
for population and the size of the dental workforce) were completed using the 2009 Department of Veteran based dental fees. ${ }^{15}$ Data was separated into the seven States and one Territory: Australian Capital Territory (ACT), Victoria (Vic), New South Wales (NSW), Queensland (QLD), Northern Territory (NT), Western Australia (WA), South Australia (SA) and Tasmania (TAS).

\section{Results}

\section{Uptake of EPDCS/CDSS}

Utilisation of the EPDCS in the first three years of implementation was significantly lower than expectation. The number of services provided during the period of 2004-2007 totalled 16,000 services. With a change in subsidy and rebranding of the scheme as the CDSS, the number of services increased exponentially. During the operation of the CDSS, the number of services were 16,297 (2007), 1,503,854 (2008) and 3,426,815 (2009). The difference between the first three years of the EPCDS and the CDSS represented an increase of $30625 \%$. Of these CDSS services, three million were provided in NSW and one million were diagnostic and an additional one million were restorative in nature (Table 1). ${ }^{13,16}$ After adjustment for the difference in population between jurisdictions, there appears to be a noticeable increase in utilisation of the scheme in NSW. Despite differences in population, the age demographics between jurisdictions are similar with no obvious differences in median age and individuals over 65 years. As an estimate of the total patients seen the number of 85,011 (comprehensive examinations) was 246,168 over the three years and peaked in 2009 at 163,626.

Time course: The number of dental services, coincident with the changes leading towards the increase in benefit and remuneration under the CDSS, showed a marked increase from 2007 (Figure 1). The value of care under the scheme in 2009 was a $24,000 \%$ increase compared to 2007 . The biggest increases were due to the increased number of fixed prosthodontic procedures, which accounted for the majority of the costs associated with the program.

Jurisdictional breakdown: The highest proportion (66\%) of services were provided in New South Wales with Victoria second (22\%) and Queensland and South Australia following with $6 \%$ each (Table 1). In 2009, two-thirds of the total value of care was provided in New South Wales (Figure 2). A comparison of the types of treatment provided shows a significant disparity between jurisdictions on a population basis. Dentists in the Northern Territory tended to rely on extraction and denture prosthodontics whilst the preferred option in NSW favoured crown, bridge and implant dentistry (Table 1, Figures 2-3). The value of care (adjusted for population discrepancies) provided as a proportion of comprehensive examinations ranged from $\$ 1937$ in the Northern Territory to $\$ 2900$ in New South Wales. The value of care per dentist ranged from nearly $\$ 80,000$ down to less than $\$ 1000$ and the value of care per adult of the population ranged between $\$ 53$ and \$1 across Australia. The highest had always been New South Wales and the lowest always being the Northern Territory (Figure 4).

Figure 1: Dental contribution to Enhanced Primary Care from 2004-2009

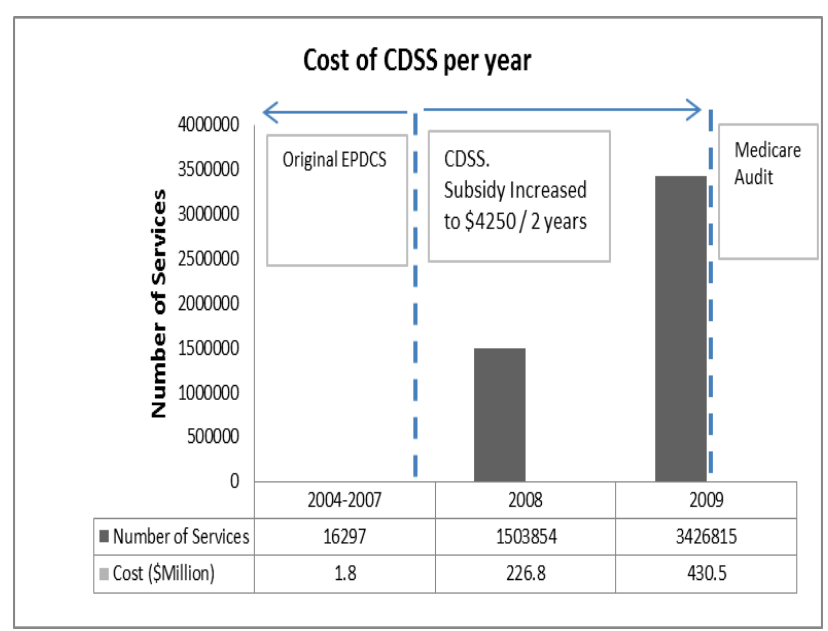

Figure 2: The proportion of value of care by item number for NSW and the rest of Australia (2009)

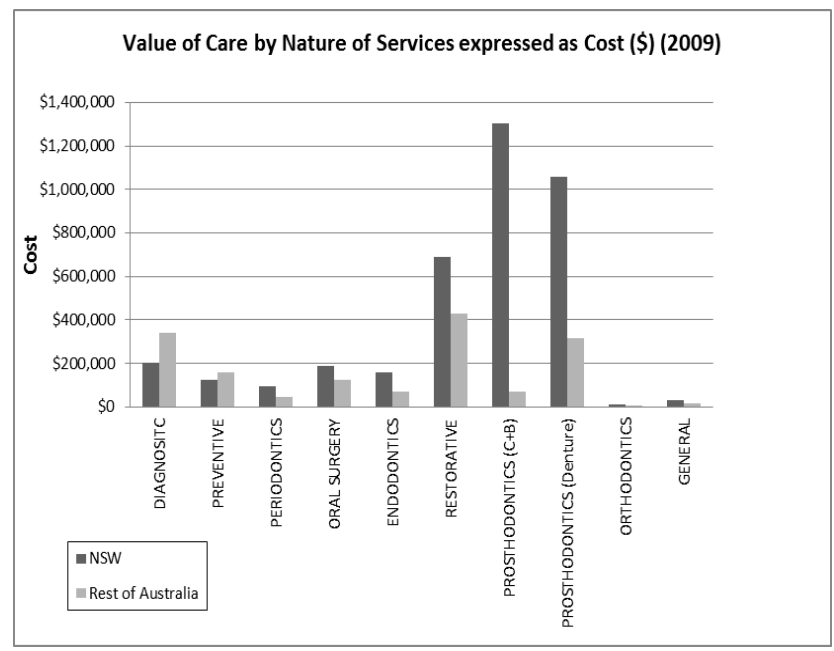


Figure 3: Cost of Value of care by type of service for NSW and rest of Australia (2009)

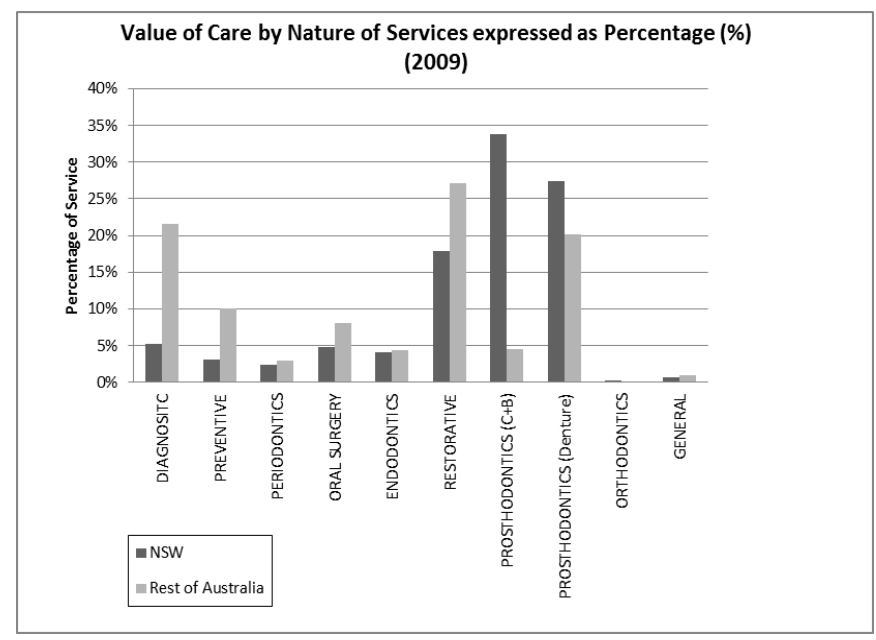

Figure 4: The average value of care per dentist (left axis -bars) and per adult (greater than $\mathbf{1 5}$ years of age) (right axis --- diamonds) for each jurisdiction of Australia

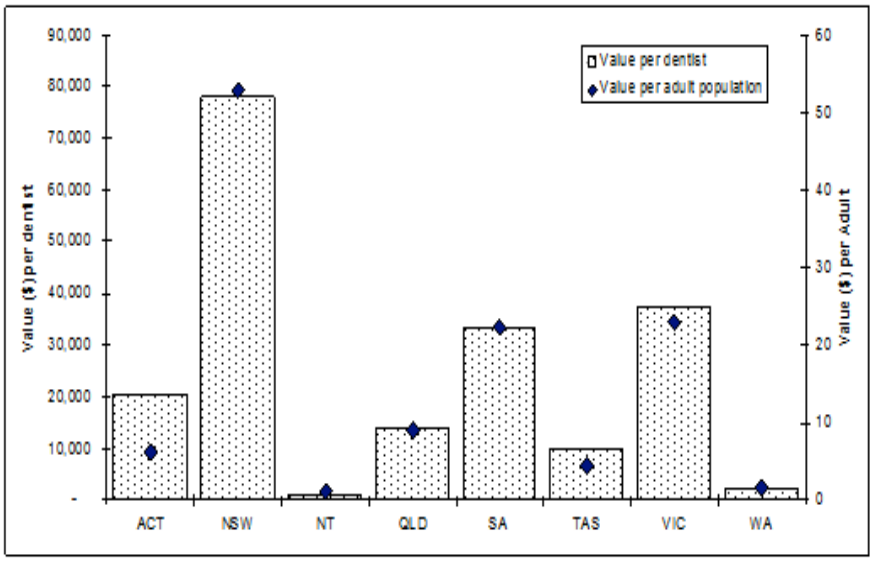

Services by sex and age: The predominant age group for services was 65--74--year-olds followed closely by the 55-64 year--old-..age group. As the impact of chronic and complex conditions tends to increase with age these findings are not surprising. ${ }^{17}$ An age demographic of the distribution of patients is shown in (Figure 5).

\section{Discussion}

A study of the CDSS provided the opportunity to observe a large national dataset of services in the vicinity of five million. As a government department with no financial incentive and with the responsibility of processing all claims, this study assumes that the collation of the data by the Department of Human Resources reflected true utilisation patterns of the scheme without bias and significant inaccuracy.
Figure 5: Age and gender of CDSS patients

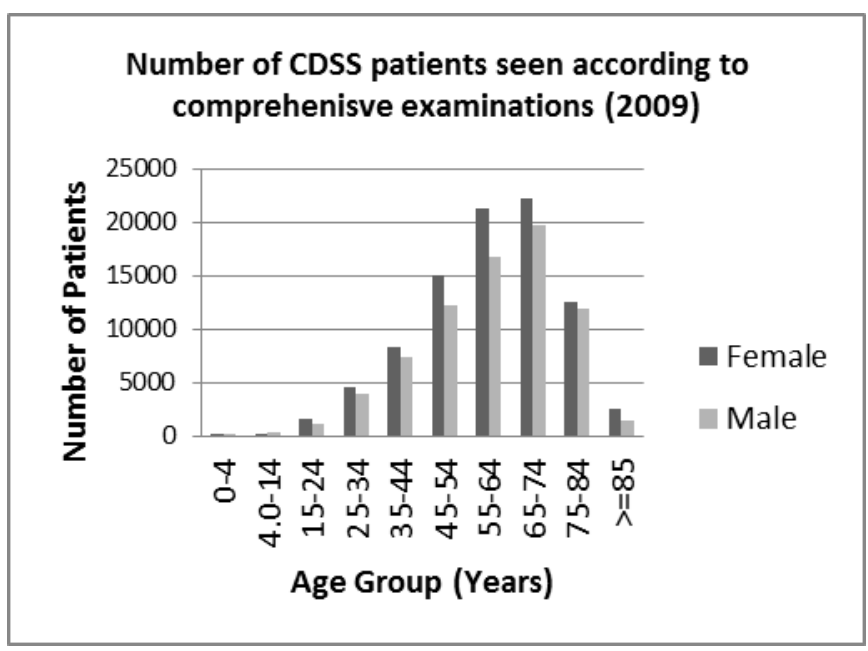

Figure 6: The proportion of items of service (grouped) provided in 2009 under the Chronic Dental Disease Scheme (CDSS) compared to previous published general practice mix of care

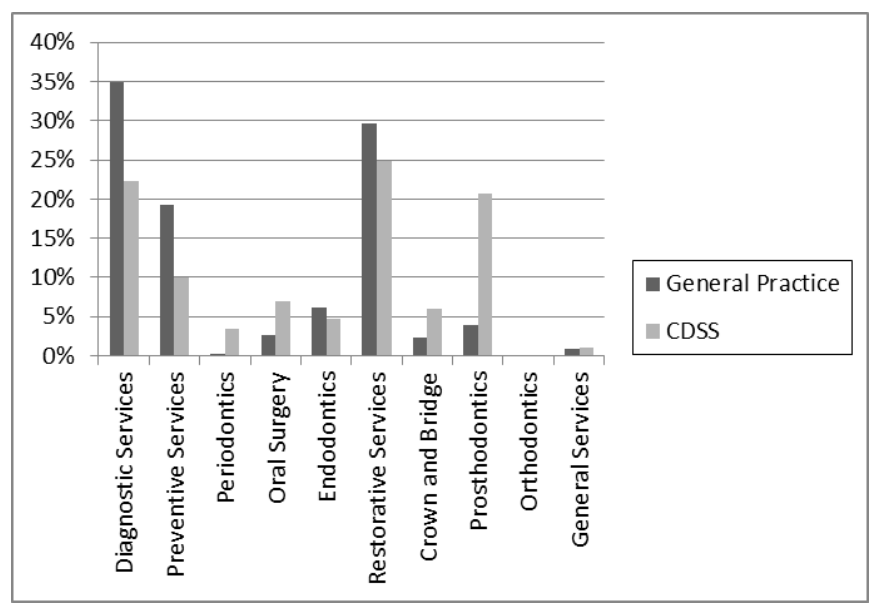

As the results of this study were obtained from open sources this study was limited by the nature of the data. Unfortunately, the dataset does not correlate the nature of services provided with the type and severity of chronic diseases. In addition, there is no indication or quantification of the extent and magnitude of patient co-contribution for service providers that opted to charge above the level of subsidy. The location of services with correlation by postcode was unobtainable and this restricted an investigation of socio-demographic status. The dataset consisted of a conglomerate of services in totality and this did not permit analysis at the patient level. It was possible that the same patient visited the dental provider on multiple occasions for a repeat course of treatment. This source of discrepancy was limited by reducing the period of 
investigation of the CDSS to a period of three years (20072009). With advances in modern dentistry and the increasing awareness of evidenced based practice, the study assumes that most patients did not require a new course of reconstructive dentistry within the period of three years. Another advantage of this three-year period is that external influences such as the animosity associated with an aggressive audit, which occurred in late 2009/10 did not influence current general practice behaviour in the provision of services under the CDSS. Another potential source of discrepancy involved the removal of specialist and prosthodontic services. This may create bias if general practitioners assumed these services without referral to prothesists or specialists. However, as dentists are accredited by the same authority in Australia (Australian Dental Council), we do not believe this to be a significant factor at the jurisdictional level whereby the variability in clinician experiences would normalise over the large dataset. Furthermore as these providers primarily see patients outside the CDSS in their private practices, the patterns of referral would be consistent for each clinician and would be indicative of general practice dentistry across Australia.

As such, a general comparison has been made with published data relating to the nature of services in general practice dentistry with CDSS patients (Figure 6). ${ }^{18,19}$ The figure shows an increase in crown and bridge prosthodontics in the CDSS scheme with obvious differences across jurisdictions. As with general practice dentistry, periodontics and orthodontics constitute very little in terms of general practice activity. It must be noted that the cohort in general practice differ to those treated under the CDSS so the aim of this comparison is to merely highlight the differences in general practice dentistry.

Despite the limitations in investigating the CDSS at the patient, disease and socio-demographic level, this study provides an interesting analysis at the jurisdictional and epidemiological level which is befitting of the nature and scope of public dental health. Although a quantitative description of specific jurisdictional factors such as the level of oral health awareness and promotion, infrastructure, resource and cultural differences were limited; this study could quantify the product of these synergistic influences through investigation of the national dataset of services for meaningful discussion.

The results of this study suggest that the utilisation of the CDSS were co-incident with changes in level of subsidisation and remuneration. Whilst the Department of Health and Aging that was responsible for the administration of the previous scheme (EPDCS) argued that the low uptake of the program was due to stakeholders "getting use to the program", the EPCDS was significantly underutilised during its entire three--year period of service ${ }^{10}$. This study postulates that low utilisation coincided with the low level of cover that resulted in significant out of pocket expenses for patients. This deterred more costly dental procedures such as crown, bridge and implant dentistry.

In contrast, the increase subsidy in the CDSS saw spectacular increases in costs primarily due to prosthodontics services in less than 12 months. Treatment plans involving more costly dental procedures were more likely to be accepted when presented to patients as financial barriers were no longer significant issues with increased government subsidisation. As the majority of chronic conditions are longstanding without cure, the significant uptake in this scheme over a short period of time tends to suggest that the financial incentives to both the clinician and patient are the driving factors in the utilisation of the scheme rather than the improvement of chronic conditions and quality of life arising from dental treatment.

One can assume with such substantial population numbers that the incidence of chronic disease would be relatively even spread across the jurisdictions. In Australia, all capital cities reside near the coast and with vast areas of rural dwellings and un-urbanised territory that are most prominent in WA, NT, QLD and SA (by surface area). The indigenous populations tended to be evenly distributed throughout the country and have the highest levels of chronic diseases in Australia. Some smaller variation in population chronic disease incidence may be present (with a focus on the Northern Territory) but it would be expected that the utilisation would be even, at the gross levels of States and Territories. Utilisation of the scheme is not relatively distributed to reflect the Australian population or patterns of chronic disease distribution. The State of NSW overwhelmingly accounted for the costs involved with the program. The choice of treatment also differed between jurisdictions. Clinicians that were aware of the program and its potential benefits have been postulated to be a significant factor in the use of the scheme, especially when eligibility criteria were very open. There have been documented cases of health service providers actively engaging their GP for referrals under the EPC schemes. ${ }^{20}$ The higher level of promotion in NSW has also influenced patient behaviour with many seeking providers that would bulk bill under the scheme to avoid out of pocket expenses. The potential for different cultures may also affect the utilisation of services. In Australia, the majority of dentists practice in the same jurisdiction where they obtained their qualification. Furthermore, collaboration between the 
dental schools at the undergraduate level is minimal and there are no nationalised standards of care.

The mix of care provided differed significantly from that reported for private dental practice. It is clearly evident that the mix was most significantly different in the high cost areas of crown and bridge and prosthodontics. Arguments can be made that the mix of care should be different to general practice but as a public health initiative targeted at reducing the effect of chronic disease on oral disease one would expect that the mix would be more towards basic removal of infection and pain reduction than towards high end care.

A neglected area in the delivery of government dental programs is one of remuneration. The current system of remuneration for both private and public dentistry in Australia is skewed towards rewarding invasive and interventive treatment, which is the source of significant cost. Preventive dentistry is poorly remunerated and difficult to implement. As general practice dentistry continues to be invasively driven, any level of subsidisation is effectively absorbed by the high costs of restorative and prosthodontic treatment. This highlights one of the deficiencies of a fee for service system of remuneration in the private system whereby financial incentive is not mutually exclusive to treatment selection. It must be emphasised that prosthodontic treatment addresses the product of disease but has no therapeutic value in disease control and stabilization which is one of the main objectives of the EPC. General dental treatment plans have an increased acceptance rate by patients as financial barriers to treatment are no longer concerns. For these reasons, regardless of the mechanics of any form and level of government subsidisation, costs would be significantly high without addressing the framework of remuneration or introducing constraints to the prosthodontic treatment options offered to patients. These considerations would be highly beneficial to future policy especially as it relates to sustainability and is an area that requires further investigation.

\section{Conclusion}

The analysis of the MBS database for dental services under the CDSS shows that it has been extensively utilised, albeit in a manner that does not necessarily reflect the distribution of chronicity as the predominant users of the scheme were patients in the geriatric age, which population statistics demonstrate were similar between jurisdictions in terms of population patterns. It has been utilised at a far higher rate in the state of NSW with prosthodontics accounting for the majority of expenses incurred. Treatment plans also differed between jurisdictions with fixed prosthodontics more common in NSW compared with other States. The increase in utilisation of the CDSS scheme was coincident with periods of increases in subsidy and remuneration and has been postulated to be a main driver for its utilisation rather than the improvement in health from dental intervention.

\section{References}

1. United Nations, Department of Economic and Social Affairs, Population Division (2011). World Population Prospects: The 2010 Revision, Volume I: Comprehensive Tables. ST/ESA/SER.A.313.

2. Australian Institute of Health and Welfare: Chronic disease and associated risk factors in Australia (AIHW cat no. PHE 81). Canberra 2006.

3. Dowrick C. The chronic disease strategy for Australia. Med J Aust. 2006 Jul 17;185(2):61-2.

4. Biggs A. Dental Reform: an overview of universal dental schemes. Department of Parliamentary Services. Australian Government; 2012.

5. Department of Health and Aging. Medicare Statistics: September quarter 2011, Table A7C. Available at http://www.health.gov.au/internet/main/publishing.nsf/con tent/medstat--sep11--tables-"-ac ( accessed March 11 2012).

6. World Health Organization. The World Health Report 2003, Continuous improvement of oral health in the 21st Century. Geneva, World Health Organization; 2003.

7. National Advisory Committee on Oral Health (NACOH). Healthy mouths, Healthy Lives: Australia's National Oral Health Plan 2004-2013, NACOH, South Australian Department of Health. 2004.

8. National Health and Hospitals Reform Commission. A healthier future for all Australians, Final Report. NHHRC. 2004.

9. Biggs A. Overview of Commonwealth involvement in funding dental care, Research Paper no.1 2008-09. Department of Parliamentary Services. Australian Government 2008.

10. Biggs A. Health Insurance Amendment (Medicare Dental Services) Bill 2007. Department of Parliamentary Services. Australian Government 2007.

11. Medicare Benefits Schedule database. Available at: http://www.medicareaustralia.gov.au/provider/medicare/m bs.shtml (accessed January 17th 2011).

12. Australian Dental Association. The Australian Schedule of Dental Services and Glossary, Ninth Edition. Australian Dental Association Inc.2009.

13. Australian Bureau of Statistics: Australian Demographic Statistics, Sept 2008 (3101.0). Canberra: Australian Bureau of Statistics; 2009. 
14. Australian Institute of Health and Welfare. Dental Workforce. Available at: http://www.aihw.gov.au/dentalworkforce (accessed 11 March 2009).

15. Department of Veterans Affairs. Fee schedule of dental services for dentists and dental specialists. Available at:

http//:www.dva.gov.au/service_providers/Fee_schedule/pa ges/dental_and_allied_health.aspx (Accessed 10 April 2012). 16. Australian Institute of Health and Welfare. Dental Workforce Statistics 2006. Australian Government. Available at: http://www.aihw.gov.au/dental--workforce (Accessed 15 June 2012).

17. Ruoff G. Challenges of managing chronic pain in the elderly. Semin Arthritis Rheum. 2002 Dec;32(3 Suppl 1):4350.

18. Brennan D, Spencer A. Trends in private dental service provision in major city and other Australian locations. Aust J Rural Health. 2007 Jun;15(3):189..95.

19. Brennan D, Spencer A. Patterns of care in private general practice by main diagnoses. Aust Dent J. 2007 Mar;52(1):67-70.

20. Haines T, Foster M, Cornwell P, Fleming J, Tweedy S, Hart $A$ et al. Impact of Enhanced Primary Care on equitable access to an economic efficiency of allied health services: a qualitative investigation. Aust Health Rev. 2010 Mar;34(1):30. -5 .

\section{PEER REVIEW}

Not commissioned. Externally peer reviewed

\section{CONFLICTS OF INTEREST}

The authors declare that they have no competing interests 


\begin{tabular}{|c|c|c|c|c|c|c|c|c|}
\hline Juris diction & NSW & VIC & QLD & SA & WA & TAS & ACT & NT \\
\hline Population ('000,000)( \%) & $6.55(33 \%)$ & $4.93(25 \%)$ & $3.9(20 \%)$ & $1.51(8 \%)$ & $1.96(10 \%)$ & $0.48(2 \%)$ & $0.32(2 \%)$ & $0.19(1 \%)$ \\
\hline Median Age & 37.2 & 36.9 & 36.2 & 39.2 & 36.2 & 39.9 & 34.6 & 31.2 \\
\hline$\%$ over 65 years of age & 14 & 14 & 13 & 16 & 12 & 16 & 11 & 6 \\
\hline Surface Area (Rank) ('000 km) & $800.6(5)$ & $227.4(6)$ & $1730.6(2)$ & $983.5(4)$ & $2529.9(1)$ & $68.4(7)$ & $2.4(8)$ & $1349.1(3)$ \\
\hline Dentist Ratio $(/ 100,000)$ & 52 & 48 & 50 & 53 & 52 & 35 & 65 & 37 \\
\hline \multicolumn{9}{|l|}{ Dental Services (Number, \%) } \\
\hline Diagnostic & $685430(68.17 \%)$ & $189245(18.82 \%)$ & $60603(6.03 \%)$ & $58202(5.79 \%)$ & $4871(0.48 \%)$ & $2798(0.28 \%)$ & 3907 (0.39\%) & $344(0.03 \%)$ \\
\hline Preventive & $148285(68.76 \%)$ & $40472(18.77 \%)$ & $12790(5.93 \%)$ & $11922(5.53 \%)$ & $854(0.4 \%)$ & $574(0.27 \%)$ & $674(0.31 \%)$ & $80(0.04 \%)$ \\
\hline Periodontics & $286682(65.34 \%)$ & $92554(21.1 \%)$ & $27737(6.32 \%)$ & $27208(6.2 \%)$ & $1466(0.33 \%)$ & $1689(0.38 \%)$ & $1295(0.3 \%)$ & $113(0.03 \%)$ \\
\hline Oral Surgery & $190603(61.20 \%)$ & $71463(22.95 \%)$ & $25089(8.06 \%)$ & $17659(5.67 \%)$ & $3012(0.97 \%)$ & $1989(0.64 \%)$ & $1396(0.45 \%)$ & $230(0.07 \%)$ \\
\hline Endodontics & $105458(67.56 \%)$ & 35168 (22.53\%) & $8252(5.29 \%)$ & $5651(3.62 \%)$ & $614(0.39 \%)$ & $295(0.19 \%)$ & $576(0.37 \%)$ & $82(0.05 \%)$ \\
\hline Restorative & $674240(62.02 \%)$ & $239993(22.08 \%)$ & $85257(7.84 \%)$ & $73910(6.8 \%)$ & $5710(0.53 \%)$ & $3209(0.3 \%)$ & $4169(0.38 \%)$ & $585(0.05 \%)$ \\
\hline Prosthodontics & $654968(67.44 \%)$ & $202448(20.85 \%)$ & $44006(4.53 \%)$ & $58490(6.02 \%)$ & $4978(0.51 \%)$ & $3227(0.33 \%)$ & $2554(0.26 \%)$ & $491(0.05 \%)$ \\
\hline Crown and Bridge & $221540(73.15 \%)$ & $54206(17.9 \%)$ & $13166(4.35 \%)$ & $10738(3.55 \%)$ & $1566(0.52 \%)$ & $790(0.26 \%)$ & $800(0.26 \%)$ & $46(0.02 \%)$ \\
\hline Orthodontics & $1535(77.64 \%)$ & $355(17.96 \%)$ & $51(2.58 \%)$ & $24(1.21 \%)$ & $4(0.20 \%)$ & $6(0.30 \%)$ & $2(0.10 \%)$ & $0(0.00 \%)$ \\
\hline General & $24371(57.73 \%)$ & $11794(27.94 \%)$ & $3389(8.03 \%)$ & $2107(4.99 \%)$ & $253(0.6 \%)$ & $158(0.37 \%)$ & $136(0.32 \%)$ & $5(0.01 \%)$ \\
\hline Total & 2993112 & 937698 & 280340 & 265911 & 23328 & 14735 & 15509 & 1976 \\
\hline
\end{tabular}

